\title{
On the Performance of Peristaltic Pumping for the MHD Slip Flow under the Variation of Elastic Walls Features
}

\author{
Ramzy M. Abumandour*, Islam M. Eldesoky, and Essam T. Abdelwahab \\ Department of Basic Eng. Sciences, Faculty of Eng., Menofia University, Egypt \\ *(Corresponding author: ramzy-0000@yahoo.com)
}

\begin{abstract}
This research is mainly concerned with studying the performance of the peristaltic flow of magnetohydrodynamic (MHD) viscous compressible flow under the impact of slip conditions, magnetic flux density, elastic features of the wave, and the liquid compressibility. The model taken represents a two-dimensional flexible sinusoidal rectangular duct with spring-damper backing. The perturbation approach is expanded as a series over the governing equations and the non-linear solution is introduced. Analytical relations describing the mean axial velocity, the mean velocity perturbation function, the net volumetric flow rate, the velocity corresponding to the elastic walls, and the critical wall tension are obtained with a small perturbation parameter known as amplitude ratio. The reversal flow occurs near the core of the channel when the critical tension is reached. The compliant wall features, magnetic flux density and slip conditions are strongly changing the dynamic behavior of the induced flow. The increase in magnetic flux causes a resisting effect to the flow and reducing the mean axial streamwise velocity and also for higher values of magnetic flux, the reverse flow occurs at the channel core. The damping property of the wall causes a reduction in the main flow but both of circumferential wall tension and the wall stiffness properties are enhancing the main flow rate. The flow of biofluids under external magnetic field through the human body is a good application related to this study such as the magnetic resonance imaging (MRI) and radiosurgery applications.
\end{abstract}

Keyword: Peristaltic flow; Wall properties; Compressible flow; MHD; Slip conditions

\section{Introduction}

The peristaltic pumping has a wide range of applications in biological and biomedical systems. Recently, the research in this area is developing rapidly and continuously due to its great importance. To be more specified, these researches are very helpful for physiologists to understand and predict what is happening for the human organs that move peristaltically under the various effects of physical properties, for instance, the magnetic flux density, the slip conditions, hall current, heat transfer, nanoparticles concentration, and the elastic muscles characteristics. To extend the view, the peristalsis occurs due to the alternation relaxation and contraction of the walls to force the content to move from one place to another. There are many real applications associated with the study of the collapsible elastic tubes. In biological systems, the peristalsis appears inside the human body in many organs as a result of collapsing compliant walls such as the internal blood hemodynamics through the cardiovascular system especially in veins above the heart, capillaries, and arteries under a cuff. The flexibility of the arteries in the real biological systems involves the muscle effect which changes the behavior of the blood flow. Experimentally, these models can be approached to reality by considering the tube law. The tube law consists of rubber tubes with finite length in the presence of self-induced vibratory oscillations. There are also many biofluids move peristaltically such as urine flow through the ureter, bolus locomotion in the digestive system, chyme transport in large and small intestines and lymph movement in the lymphatic tract. Peristaltic principles are used in biomedical engineering to produce a heart-lung pump which helps to pump the blood through the body and lungs during open-heart surgery. On the other hand, in petroleum engineering, the mechanical peristaltic pump is used to extract the crude oil from the porous rock.

The peristaltic pumping is handled in many articles since Latham [1] who did the first attempt to analyze the nature of fluid motion using the principles of the peristaltic. Many articles [2-14] were concentrated on studying the impact of various physical properties on the peristaltic action of incompressible liquids. The slip flow occurs when the particles don't stick with the wall and there is a relative velocity at the boundaries, but the no-slip conditions appear when the particles of fluid adhere to the walls, and so the fluid velocity is zero relative to the wall. The effect of slip and no-slip conditions was taken into consideration in the studies of $[7,15,16]$.

The magnetohydrodynamics (MHD) phenomena result from the mutual effect of a magnetic field and a conducting fluid flowing across it. Sud et al. [14] analyzed the influence of magnetic flux on the increase of the blood velocity. Akbar [17] studied the action of the transverse magnetic field on the peristaltic flow of nano-EyringPowell fluid. Hayat et al. [18] used the long-wavelength technique to study the influence of electromagnetic force resulting from the axial magnetic field on the pressure gradient and stream function of the peristaltic flow. Abbasi et al. [19] showed the impact of the variable viscosity and 
the magnetic flux about the origin on the net flux and net axial velocity.

For the compressible flow point of view, Mekheimer el al. [20] study was concerned with the combined effects of magnetic intensity, the permeability of porous medium and the slip conditions on a compliant peristaltic wave of a driven Maxwellian fluid through a microchannel. Recent articles [15, 21-26] involve the peristaltic flow of MHD under various physical parameters such as nanoparticle concentration, Hall current intensity, the permeability of porous medium and wall slip coefficient are presented. The effectiveness of liquid compressibility within the peristaltic pumping was explained under an ultrasonic system by Aarts and Ooms [27]. It was found that the varying compressibility factor results in changing the fluid flow dynamic behavior. Eldesoky and Mousa [28, 29] introduced the combined influences of the liquid compressibility, relaxation time, porosity of porous medium and slip conditions on the net flux and the reversal flow through tapered pore. Whereas, Eldesoky et al. [30] were interested in knowing the nature of flow when suspended particles exist within the driven peristaltic two-phase flow under the perturbation approach. This idea is very useful to approach the real application system as it occurs in the case of blood clots and kidney stones move through the ureter. They found that the increase in particulate concentration results in a decrease in the main flow and an increase in the reversed pressure and reversal flow. Abumandour et al. [31] in their recent study did a complementary study to Eldesoky et al. [30] as they added the slip conditions at the boundaries through the two-phase flow.

Compliance is the ability of the conduit walls to expand and accommodate the increased content. The walls of biological organs are elastic and extensible. The force corresponding to walls appears as a result of interaction between the muscle forces, internal flow, and external wall forces. Choosing the compliant walls is very important to indicate its effectiveness in the flow behavior in the critical collapsible organs in the human body. The flexible walls features are wall stiffness, damping, tension, and wall bending factor. Pandey and Chaube [32] studied the interaction of wall flexibility with the peristaltic locomotion of couple stress fluid. Javed et al. [33] introduced the effect of the compliant walls on the flow of non-Newtonian fluid. Elnaby and Haroun [34] expressed the wall elasticity by using a new compliant model and showed that net flux and mean velocity have been changed at different values of wall tension, and damping. Eldesoky et al. [35] were mainly interested in showing the different effects of wall flexibility, slip conditions, liquid compressibility and relaxation time on the peristalsis of Maxwellian compressible flow. The results showed that all the important physical properties have participated in changing the characteristics of the flowing flow. Whereas, Eldesoky et al. [36] added the effect of heat transfer which is very helpful in the case of cancer disease treatment and showed that the wall elasticity and wall slip made a mutation in the net flow behavior. Mekheimer and AbdelWahab [37] investigated the combined effects of slip compliant boundaries of a microchannel and liquid compressibility on the physical fluid properties of the induced surface acoustic wave. Recently, there is a great concentration on this point of study. In Sadaf and Abdelsalam [38] the performance of the injected nanoparticles is investigated in a mixed flexible peristaltic blood model using average lubrication methodology. It was found that hybrid nanoparticles were affecting the heat transfer rate rather than the set nanoparticles. Bhatti et al. [39] have investigated the Sutterby fluid model to understand the mechanism of the blood hemodynamics under the effect of nanomaterials characteristics on the runoff of the gyrotactic microorganisms through the blood in a norrow artery. The accurate perturbation technique up to the third order was performed on the model's governing equations. And the results showed that non-Newtonian property has a resisting impact and the temperature distribution was high while transition from convergent position to divergent one through the artery. Abdelsalam and Bhatti [40] have analyzed also the peristaltic locomotion of non-Newtonian nanofluid convective flow in the presence of heat transfer and carrying some oxytocic microorganisms using the homotopy perturbation methodology (HPM). Elmaboud et al. [41] have entered the electromagnetic force field and the heat flux on the wavy flow of two layer immiscible flow. The analytical methodology is the homotopy analysis method (HAM). The results showed that the electric flied boosts the net flow whereas the magnetic force did not. Abdelsalam and Mekheimer [42] have investigated the case of rotating channel with constant angular velocity and carrying couple stress fluid moving peristaltically under the exact solution of (HPM). Eldesoky et al. [43] have combined the mutual effects of thermal heating and magnetic flux in the presence of small circular suspensions through the blood flow in a catheter tube under the approximation of the long wave technique. The results showed that the heat was enhancing the net axial velocity while the suspension concentration caused a reduction in the flow rate. While Eldesoky et al. [44] added the porosity effect to the Magnetohydrodynamics (MHD), heating, and dusty suspensions properties on the peristaltic flow of the blood via a two dimensional channel under the assumptions of perturbation scheme. The more dust leaded to more decrease in the fluid temperature and also the magnetic force has changed the fluid temperature depending on the time and position. Many recent articles [45-49] were interested in the peristaltic mechanism under the different effects of heat and mass transfer, magnetic flux, suspensions, nanoparticles concentration, entropy generation and the behavior of the tumor cells under the magnetism in the presence of the peristalsis for both compressible and incompressible flow and different flow models such as Maxwellian, Bingham models using various analytical and numerical methods.

From the comprehensive survey, it is noted that no previous attempt has combined the influences of magnetic flux density, compliant wall features, wall slip conditions and liquid compressibility on the compressible peristaltic transport through a two-dimensional channel. Therefore, 
the authors are interested in this study because of its importance in the biomedical field. It is can be found in the real-life applications such as the radiosurgery and magnetic resonance radiation on the human body. The problem model is considered to be a sinusoidal elastic wall with spring-damper backing. The perturbation methodology with a small perturbation parameter called amplitude ratio is introduced. The flow is considered to be a free pumping case by neglecting the pressure gradient in the beginning. The chosen model approaches the real systems in case of exposing the human body to magnetic field radiation. And the current study is a special case related with that of Mekheimer et al [37] if the magnetic effect is neglected.

\section{The problem formulation}

\section{Problem Geometry}

The undertaken model is supposed to be a twodimensional elastic sinusoidal channel with spring-damper backing in the presence of the induced transverse magnetic field, See Fig. 1.

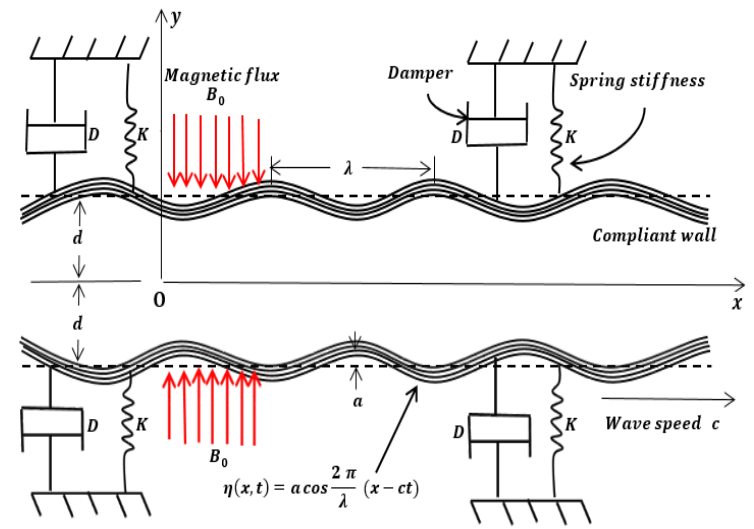

Figure 1- The peristaltic geometry with spring-damper backing

\section{Mathematical Formulation}

Governing equations

The basic system of equations (mass and momentum conservation) and equation of state for compressible flow in presence of magnetic field are expressed as [20, 27]:

Continuity equation

$\frac{\partial \rho}{\partial t}+\nabla \cdot(\rho \overrightarrow{V)}=0$

Momentum equation

$\rho \frac{\partial \vec{V}}{\partial t}+\rho(\vec{V} \cdot \nabla) \vec{V}=-\nabla \vec{p}+\mu\left(\nabla^{2} \vec{V}\right)+\frac{\mu}{3} \nabla(\nabla \cdot \vec{V})-\vec{F}$

Equation of state

$$
\begin{aligned}
& \frac{\partial \rho}{\partial p}=\rho k^{*} \\
& \rho=\rho_{o} e^{k^{*}\left(p-p_{c}\right)}
\end{aligned}
$$

The compliant wall geometry is given by:

$\eta(x, t)=a \cos \frac{2 \pi}{\lambda}(x-c t)$

It is defined at the upper and lower wall as follows:

$$
y= \pm(d+\eta)= \pm\left(d+a \cos \frac{2 \pi}{\lambda}(x-c t)\right)
$$

The elastic wall equation is expressed as [37]:

$L(\eta)=p-p_{o}=-T \frac{\partial^{2} \eta}{\partial x^{2}}+m \frac{\partial^{2} \eta}{\partial t^{2}}+D \frac{\partial \eta}{\partial t}+B \frac{\partial^{4} \eta}{\partial x^{4}}+K \eta$

The electromagnetic force and the velocity vector are expressed by:

$$
\begin{aligned}
& \vec{F}=\sigma u B_{o}{ }^{2} \hat{i} \\
& \vec{V}=u \hat{i}+v \hat{j}
\end{aligned}
$$

\section{Boundary conditions}

The walls of the channel are controlling the shape and the dynamical properties of the flow. The main characteristics corresponding to the boundaries $y= \pm(d+\eta)$ are the parameter of interest as follows:

Wall slip:

$u=\mp A \frac{\partial u}{\partial y}$

Wall impermeability:

$v= \pm \frac{\partial \eta}{\partial t}$

The surface wall interaction forces are:

$\frac{\partial L}{\partial x}=\frac{\partial P}{\partial x}=-\rho\left(\frac{\partial u}{\partial t}+u \frac{\partial u}{\partial x}+v \frac{\partial u}{\partial x}\right)+\mu\left(\frac{\partial^{2} u}{\partial x^{2}}+\frac{\partial^{2} u}{\partial y^{2}}\right)+\frac{\mu}{3} \frac{\partial}{\partial x}\left(\frac{\partial u}{\partial x}+\frac{\partial v}{\partial y}\right)-\sigma u B_{o}^{2}$

\section{Solution methodology}

Firstly, non-dimensional analysis is done for both governing equations and boundary conditions. Secondly, the perturbation series is expanded over the resulting dimensionless governing equations and the boundary conditions. Finally, the non-linear approximation is presented for the resulting relations. Taylor series expansions are used to simplify the boundary conditions.

\section{Non-dimensional analysis}

Dimensionless flow parameters are: 
$\bar{x}=\frac{x}{d}, \bar{y}=\frac{y}{d}, \bar{u}=\frac{u}{c}, \bar{v}=\frac{v}{c}, \bar{\eta}=\frac{\eta}{d}, \bar{t}=\frac{c t}{d}, \bar{p}=\frac{P}{\rho_{o} c^{2}}, \overline{p_{c}}=\frac{P_{c}}{\rho_{o} c^{2}}$

Dimensionless elastic wall parameters are:

$\bar{D}=\frac{D d}{\rho_{o} v}, \quad \bar{B}=\frac{B}{\rho_{o} d v^{2}}, \quad \bar{K}=\frac{K d^{3}}{\rho_{o} v^{2}}, \quad \bar{m}=\frac{m}{\rho_{o} d}, \quad \bar{T}=\frac{T d}{\rho_{o} v^{2}}$

Dimensionless system of equations are:

$\frac{\partial \rho}{\partial t}+u \frac{\partial \rho}{\partial x}+v \frac{\partial \rho}{\partial y}+\rho\left(\frac{\partial u}{\partial x}+\frac{\partial v}{\partial y}\right)=0$

$\rho\left[\frac{\partial u}{\partial t}+u \frac{\partial u}{\partial x}+v \frac{\partial u}{\partial y}\right]=-\frac{\partial p}{\partial x}+\frac{1}{R}\left[\frac{\partial^{2} u}{\partial x^{2}}+\frac{\partial^{2} u}{\partial y^{2}}\right]+\frac{1}{3 R} \frac{\partial}{\partial x}\left[\frac{\partial u}{\partial x}+\frac{\partial v}{\partial y}\right]+-\frac{H_{a}^{2}}{R} u$

$\rho\left[\frac{\partial v}{\partial t}+u \frac{\partial v}{\partial x}+v \frac{\partial v}{\partial y}\right]=-\frac{\partial p}{\partial y}+\frac{1}{R}\left[\frac{\partial^{2} v}{\partial x^{2}}+\frac{\partial^{2} v}{\partial y^{2}}\right]+\frac{1}{3 R} \frac{\partial}{\partial y}\left[\frac{\partial u}{\partial x}+\frac{\partial v}{\partial y}\right]$

$\rho=e^{\chi\left(p-p_{0}\right)}$

Dimensionless boundary conditions at $y= \pm(1+\eta)$ with $\eta=\varepsilon \alpha \cos (x-t)$ are

$u=\mp K n \frac{\partial u}{\partial y}$

$v= \pm \varepsilon \alpha \sin \alpha(x-t)$

$\frac{\partial}{\partial x}\left(m \frac{\partial^{2}}{\partial t^{2}}+\frac{D}{R} \frac{\partial}{\partial t}+\frac{B}{R^{2}} \frac{\partial^{4}}{\partial x^{4}}-\frac{T}{R^{2}} \frac{\partial^{2}}{\partial x^{2}}+\frac{K}{R^{2}}\right) \eta=-\rho\left(\frac{\partial u}{\partial t}+u \frac{\partial u}{\partial x}+v \frac{\partial u}{\partial x}\right)+\frac{1}{R}\left(\frac{\partial^{2} u}{\partial x^{2}}+\frac{\partial^{2} u}{\partial y^{2}}\right)$

$+\frac{1}{3 R} \frac{\partial}{\partial x}\left(\frac{\partial u}{\partial x}+\frac{\partial v}{\partial y}\right)-\frac{H_{a}^{2}}{R} u$

Perturbation series

Expanding the properties of the fluid as perturbation series with small amplitude ratio $(\varepsilon)$ as following

$\left.\begin{array}{l}p=p_{0}+\varepsilon p_{1}(x, y, t)+\varepsilon^{2} p_{2}(x, y, t)+\cdots \\ u=\varepsilon u_{1}(x, y, t)+\varepsilon^{2} u_{2}(x, y, t)+\cdots \\ v=\varepsilon v_{1}(x, y, t)+\varepsilon^{2} v_{2}(x, y, t)+\cdots \\ \rho=1+\varepsilon \rho_{1}(x, y, t)+\varepsilon^{2} \rho_{2}(x, y, t)+\cdots\end{array}\right\}$

Free pumping case means $\left(u_{o}\right.$ and $\left.v_{o}=0\right)$.

Two systems of equations are obtained in terms of $\varepsilon$ and $\varepsilon^{2}$ as a result of substituting Eq. 21 into Eqs. (15-18).

The system of $\varepsilon$ is

$\frac{\partial u_{1}}{\partial t}+\frac{\partial u_{1}}{\partial x}+\frac{\partial v_{1}}{\partial y}=0$

$\frac{\partial u_{1}}{\partial t}=-\frac{\partial p_{1}}{\partial x}+\frac{1}{R}\left(\frac{\partial^{2} u_{f 1}}{\partial x^{2}}+\frac{\partial^{2} u_{f 1}}{\partial y^{2}}\right)+\frac{1}{3 R} \frac{\partial}{\partial x}\left(\frac{\partial u_{1}}{\partial x}+\frac{\partial v_{1}}{\partial y}\right)-\frac{H_{a}^{2}}{R} u_{1}$

$\frac{\partial v_{1}}{\partial t}=-\frac{\partial p_{1}}{\partial y}+\frac{1}{R}\left(\frac{\partial^{2} v_{1}}{\partial x^{2}}+\frac{\partial^{2} v_{1}}{\partial y^{2}}\right)+\frac{1}{3 R} \frac{\partial}{\partial y}\left(\frac{\partial u_{1}}{\partial x}+\frac{\partial v_{1}}{\partial y}\right)$

$\rho_{1}=\chi p_{1}$

The system of $\varepsilon^{2}$ is

$\frac{\partial u_{2}}{\partial t}+u_{1} \frac{\partial \rho_{1}}{\partial x}+v_{1} \frac{\partial \rho_{1}}{\partial y}+\frac{\partial u_{2}}{\partial x}+\frac{\partial v_{2}}{\partial y}+\rho_{1}\left(\frac{\partial u_{1}}{\partial x}+\frac{\partial v_{1}}{\partial y}\right)=0$

$\left[\frac{\partial u_{2}}{\partial t}+u_{1} \frac{\partial u_{1}}{\partial x}+v_{1} \frac{\partial u_{1}}{\partial y}+\rho_{1} \frac{\partial u_{1}}{\partial t}\right]=-\frac{\partial p_{2}}{\partial x}+\frac{1}{R}\left(\frac{\partial^{2} u_{2}}{\partial x^{2}}+\frac{\partial^{2} u_{2}}{\partial y^{2}}\right)+\frac{1}{3 R} \frac{\partial}{\partial x}\left(\frac{\partial u_{2}}{\partial x}+\frac{\partial v_{2}}{\partial y}\right)-\frac{H_{a}^{2}}{R} u_{2}$ 
$\left[\frac{\partial v_{f 2}}{\partial t}+u_{1} \frac{\partial v_{1}}{\partial x}+v_{1} \frac{\partial v_{1}}{\partial y}+\rho_{1} \frac{\partial v_{1}}{\partial t}\right]=-\frac{\partial p_{2}}{\partial y}+\frac{1}{R}\left(\frac{\partial^{2} v_{2}}{\partial x^{2}}+\frac{\partial^{2} v_{2}}{\partial y^{2}}\right)+\frac{1}{3 R} \frac{\partial}{\partial y}\left(\frac{\partial u_{2}}{\partial x}+\frac{\partial v_{2}}{\partial y}\right)$

$\rho_{2}=\chi p_{2}+\frac{\chi^{2} p_{1}^{2}}{2}$

Taylor series expansion about $\mathrm{y}= \pm 1$ is given by

$$
\left.\begin{array}{l}
u( \pm \eta)=u( \pm 1) \pm \eta(x, t) \frac{\partial u( \pm 1)}{\partial y}+\frac{\eta^{2}(x, t)}{2 !} \frac{\partial^{2} u( \pm 1)}{\partial y^{2}}+\ldots \\
v( \pm \eta)=v( \pm 1) \pm \eta(x, t) \frac{\partial v( \pm 1)}{\partial y}+\frac{\eta^{2}(x, t)}{2 !} \frac{\partial^{2} v( \pm 1)}{\partial y^{2}}+\ldots
\end{array}\right\}
$$

Substituting perturbation series (21) and Taylor expansions (30) into the boundary conditions

(19) and (20) two sets of equations are obtained. The $\varepsilon$ set is

$u_{1}( \pm 1)=\mp K n \frac{\partial u_{1}( \pm 1)}{\partial y}$

$v_{1}( \pm 1)=\mp \frac{i \alpha}{2}\left(e^{i \alpha(x-t)}-e^{-i \alpha(x-t)}\right)$

$-R \frac{\partial u_{1}( \pm 1)}{\partial t}+\frac{1}{R}\left(\frac{\partial^{2} u_{1}( \pm 1)}{\partial x^{2}}+\frac{\partial^{2} u_{1}( \pm 1)}{\partial y^{2}}\right)+\frac{1}{3 R} \frac{\partial}{\partial x}\left(\frac{\partial u_{1}( \pm 1)}{\partial x}+\frac{\partial v_{1}( \pm 1)}{\partial y}\right)-H_{a}^{2} u_{1}( \pm 1)$

$=\left[-\frac{i \delta_{a}}{2}\left(e^{i \alpha(x-t)}-e^{-i \alpha(x-t)}\right)+\frac{\delta_{b}}{2}\left(e^{i \alpha(x-t)}+e^{-i \alpha(x-t)}\right)\right]$

The $\varepsilon^{2}$ set is

$u_{2}( \pm 1) \pm \frac{1}{2}\left(e^{i \alpha(x-t)}+e^{-i \alpha(x-t)}\right) \frac{\partial u_{1}( \pm 1)}{\partial y}=\mp K n \frac{\partial u_{2}( \pm 1)}{\partial y}-\frac{K n}{2}\left(e^{i \alpha(x-t)}+e^{-i \alpha(x-t)}\right) \frac{\partial^{2} u_{1}( \pm 1)}{\partial y^{2}}$

$v_{2}( \pm 1) \pm \frac{1}{2}\left(e^{i \alpha(x-t)}+e^{-i \alpha(x-t)}\right) \frac{\partial v_{1}( \pm 1)}{\partial y}=0$

\section{The Nonlinear solution}

Using the non-linear expressions of [27] to simplify the solution as follows:

$u_{1}(x, y, t)=U_{1}(y) e^{i \alpha(x-t)}+\bar{U}_{1}(y) e^{-i \alpha(x-t)}$

$v_{1}(x, y, t)=V_{1}(y) e^{i \alpha(x-t)}+\bar{V}_{1}(y) e^{-i \alpha(x-t)}$

$p_{1}(x, y, t)=P_{1}(y) e^{i \alpha(x-t)}+\bar{P}_{1}(y) e^{-i \alpha(x-t)}$

$\rho_{1}(x, y, t)=\chi P_{1}(y) e^{i \alpha(x-t)}+\chi \bar{P}_{1}(y) e^{-i \alpha(x-t)}$

The second order non-linear approach is given by

$$
\begin{aligned}
& u_{2}(x, y, t)=U_{20}(y)+U_{2}(y) e^{2 i \alpha(x-t)}+\bar{U}_{2}(y) e^{-2 i \alpha(x-t)} \\
& v_{2}(x, y, t)=V_{20}(y)+V_{2}(y) e^{2 i \alpha(x-t)}+\bar{V}_{2}(y) e^{-2 i \alpha(x-t)} \\
& p_{2}(x, y, t)=P_{20}(y)+P_{2}(y) e^{2 i \alpha(x-t)}+\bar{P}_{2}(y) e^{-2 i \alpha(x-t)} \\
& \rho_{2}(x, y, t)=D_{20}(y)+D_{2}(y) e^{2 i \alpha(x-t)}+\bar{D}_{2}(y) e^{-2 i \alpha(x-t)}
\end{aligned}
$$

Applying the nonlinear approach (36) and (37) in relations. (22-29) and their boundary conditions (31 -35) resulting in the following two sets of differential equations. The first-order set is $V_{1}^{\prime}+i \alpha U_{1}=i \alpha \chi P_{1}$

$-i \alpha R U_{1}=-i \alpha R P_{1}+U_{1}^{\prime \prime}-\alpha^{2} U_{1}+\frac{1}{3} i \alpha\left[V_{1}^{\prime}+i \alpha U_{1}\right]-H_{a}^{2} U_{1}$

$-i \alpha R U_{1}-i \alpha R V_{1}=-R P_{1}^{\prime}+V_{1}^{\prime \prime}-\alpha^{2} V_{1}+\frac{1}{3} \frac{d}{d y}\left[V_{1}^{\prime}+i \alpha U_{1}\right]$ 
Under the following boundary conditions

$U_{1}( \pm 1)=\mp K n \frac{\partial U_{1}( \pm 1)}{\partial y}$

$V_{1}( \pm 1)=\mp \frac{i \alpha}{2}$

$\frac{\partial^{2} U_{1}( \pm 1)}{\partial y^{2}}-\left[\frac{4}{3} \alpha^{2}-i \alpha R+H_{a}^{2}\right] U_{1}( \pm 1)+\frac{i \alpha}{3} \frac{\partial V_{1}( \pm 1)}{\partial y}=R \delta$

Following the same steps of the methodology used in [37] results in the equations of velocity and pressure as follows:

$U_{1}(y)=R_{6} C_{1} \cosh \left(L_{1} y\right)+R_{7} C_{2} \cosh \left(L_{2} y\right)$

$V_{1}(y)=C_{1} \sinh \left(L_{1} y\right)+C_{2} \sinh \left(L_{2} y\right)$

$P_{1}(y)=R_{4} C_{1} \cosh \left(L_{1} y\right)+R_{5} C_{2} \cosh \left(L_{2} y\right)$

The second-order set is

$V_{20}^{\prime}=-\chi\left[P_{1} \overline{V_{1}^{\prime}}+\overline{P_{1}} V_{1}^{\prime}+V_{1} \overline{P_{1}^{\prime}}+\overline{V_{1}} P_{1}^{\prime}\right]$

$U_{20}{ }^{\prime \prime}=R\left[i \alpha \chi P_{1} \overline{U_{1}}-i \alpha \chi \overline{P_{1}} U_{1}+V_{1} \overline{U_{1}^{\prime}}+\overline{V_{1}} U_{1}^{\prime}\right]+H_{a}^{2} U_{20}$

$-R P_{20}^{\prime}+\frac{4}{3} V_{20}^{\prime \prime}=R\left[i \alpha \chi P_{1} \bar{V}_{1}-i \alpha \chi \bar{P}_{1} V_{1}-i \alpha U_{1} \bar{V}_{1}+i \alpha \overline{U_{1} V_{1}}+V_{1} \bar{V}_{1}^{\prime}+\overline{V_{1} V_{1}{ }^{\prime}}\right]$

Under the following boundary conditions

$U_{2 o}( \pm 1) \pm \frac{1}{2}\left({\overline{U_{1}}}^{\prime}( \pm 1)+U_{1}^{\prime}( \pm 1)\right)=\mp K n\left[U_{2 o}^{\prime}( \pm 1) \pm \frac{1}{2}\left({\overline{U_{1}}}^{\prime \prime}( \pm 1)+U_{1}^{\prime \prime}( \pm 1)\right)\right]$

$V_{20}( \pm 1) \pm \frac{1}{2}\left[V_{1}^{\prime}( \pm 1)+\overline{V_{1}^{\prime}}( \pm 1)\right]=0$

The solution of the second-order equations (46-48) under their corresponding boundary conditions (49) and (50) is given by

$U_{2 o}(y)=R E(y)+D_{2} \operatorname{Cosh} \sqrt{\delta_{c}} y+D_{3} \operatorname{Sinh} \sqrt{\delta_{c}} y$

$V_{20}(y)=-\chi\left[P_{1}(y) \overline{V_{1}}(y)+\overline{P_{1}}(y) V_{1}(y)\right]$

$E(y)=\left[\frac{\delta_{1}}{2}\left(\frac{\cosh \left(L_{1}+\overline{L_{1}}\right) y}{\left(L_{1}+\overline{L_{1}}\right)^{2}-\delta_{c}}+\frac{\cosh \left(L_{1}-\overline{L_{1}}\right) y}{\left(L_{1}-\overline{L_{1}}\right)^{2}-\delta_{c}}\right)+\frac{\delta_{2}}{2}\left(\frac{\cosh \left(L_{1}+\overline{L_{2}}\right) y}{\left(L_{1}+\overline{L_{2}}\right)^{2}-\delta_{c}}+\frac{\cosh \left(L_{1}-\overline{L_{2}}\right) y}{\left(L_{1}-\overline{L_{2}}\right)^{2}-\delta_{c}}\right)\right.$

$+\frac{\delta_{3}}{2}\left(\frac{\cosh \left(L_{2}+\overline{L_{1}}\right) y}{\left(L_{2}+\overline{L_{1}}\right)^{2}-\delta_{c}}+\frac{\cosh \left(L_{2}-\overline{L_{1}}\right) y}{\left(L_{2}-\overline{L_{1}}\right)^{2}-\delta_{c}}\right)+\frac{\delta_{4}}{2}\left(\frac{\cosh \left(L_{2}+\overline{L_{2}}\right) y}{\left(L_{2}+\overline{L_{2}}\right)^{2}-\delta_{c}}+\frac{\cosh \left(L_{2}-\overline{L_{2}}\right) y}{\left(L_{2}-\overline{L_{2}}\right)^{2}-\delta_{c}}\right)$

$+\frac{\delta_{5}}{2}\left(\frac{\cosh \left(L_{1}+\overline{L_{1}}\right) y}{\left(L_{1}+\overline{L_{1}}\right)^{2}-\delta_{c}}-\frac{\cosh \left(L_{1}-\overline{L_{1}}\right) y}{\left(L_{1}-\overline{L_{1}}\right)^{2}-\delta_{c}}\right)+\frac{\delta_{6}}{2}\left(\frac{\cosh \left(L_{2}+\overline{L_{1}}\right) y}{\left(L_{2}+\overline{L_{1}}\right)^{2}-\delta_{c}}-\frac{\cosh \left(L_{2}-\overline{L_{1}}\right) y}{\left(L_{2}-\overline{L_{1}}\right)^{2}-\delta_{c}}\right)$

$\left.+\frac{\delta_{7}}{2}\left(\frac{\cosh \left(L_{1}+\overline{L_{2}}\right) y}{\left(L_{1}+\overline{L_{2}}\right)^{2}-\delta_{c}}-\frac{\cosh \left(L_{1}-\overline{L_{2}}\right) y}{\left(L_{1}-\overline{L_{2}}\right)^{2}-\delta_{c}}\right)+\frac{\delta_{8}}{2}\left(\frac{\cosh \left(L_{2}+\overline{L_{2}}\right) y}{\left(L_{2}+\overline{L_{2}}\right)^{2}-\delta_{c}}-\frac{\cosh \left(L_{2}-\overline{L_{2}}\right) y}{\left(L_{2}-\overline{L_{2}}\right)^{2}-\delta_{c}}\right)\right]$

The dynamic behavior of the flow is analyzed utilizing the net axial velocity, the net flow rate, the mean velocity perturbation function and the critical wall tension which is similar in effect to the pressure gradient at which reversal flow occurs. All these relations are obtained as follows:

$$
\begin{aligned}
& <V_{x}(y)>=\varepsilon^{2} U_{20}(y) \\
& G(y)=-\frac{200}{\alpha^{2} R^{2}}(E(y)-E(1)) \\
& <Q>=\varepsilon^{2} \int_{0}^{1} U_{20}(y) \mathrm{dy}
\end{aligned}
$$

The critical values of wall tension occur corresponding to the reflux conditions when 
$<V_{x}(0)>=0$

\section{Graphical Results and discussion}

Verification of analytical method

Checking and validating the results of the work requires a comparative analysis with the previous studies and the results of Mekheimer et al [37] is achieved for the present study in the absence of magnetic flux effect $\mathrm{Ha}=0$. For instance, Figure (2- a, b, and c) (corresponding to Table (1)) are obtained when $\mathrm{Ha}=0$ which are exactly coincident with that of Mekheimer et al [37].

\section{Results and discussion}

The alternation of magnetic flux density, compliant walls characteristics and flow properties have obvious effectiveness in changing the dynamical behavior of the flowing flow. This is represented in the following section. Figure (3) (corresponding to Table (2)) shows the various effects of wall damping, wall stiffness, wall tension, wall slip, magnetic flux, and liquid compressibility on the net axial velocity. Figure 3(a) shows the resisting action of the wall damping on the motion.it is also noted that the wall tension, spring stiffness and slip at walls are enhancing the net flux as the resulting increase in mean axial velocity according to the increase in the spring stiffness $(\boldsymbol{K})$, longitude wall tension $(\boldsymbol{T})$ and Knudsen number $(\boldsymbol{K} \boldsymbol{n})$, see Figures 3(b, c, and d). Figure 3(e) shows the reduction of the mean velocity due to the increase of magnetic flux density since the reversal flow begins to appear near the walls when the magnetic factor $(\boldsymbol{H a}=\mathbf{4})$ and the reverse effect increases near the boundaries at high values of magnetic flux. The liquid compressibility $(\chi)$ is inversely proportional to the net streamwise velocity so it causes the mean flow flux to decrease and this action is illustrated in Figure 3(f). The elastic compliant walls properties and wall slip are highly affecting the velocity distribution, especially at the boundaries, so that it is important to get close to the boundaries to analyze the dynamic nature of the flow and to determine the conditions at which the reversal flow will occur. The next section is related with the analysis of the velocity at the boundaries $\left(\boldsymbol{D}_{\text {wall }}\right)$ under the varying values of magnetic flux factor $(\boldsymbol{H} \boldsymbol{a})$, the wall Knudsen number $(\boldsymbol{K} \boldsymbol{n})$, the wall damping factor $(\boldsymbol{D})$, the longitude wall tension $(\boldsymbol{T})$ and the fluid compressibility $(\chi)$. All these effects are shown in Fig. (4) (corresponding to Table (3)). The analysis of the streamwise velocity at the elastic channel walls draws the interest to explain what exactly exists and happens to the behavior of the flow near the walls under the change in elastic wall features and magnetic flux intensity. It is shown in Figure 4(a) that the increase in bending coefficient $(\boldsymbol{B})$ boosts the streamwise velocity at the channel walls $\left(\boldsymbol{D}_{\text {wall }}\right)$. The influence of wall tension $(\boldsymbol{T})$ and stiffness $(\boldsymbol{K})$ is illustrated in Figures $4(\mathrm{~d}$, and $\mathrm{f})$ and it is noted that $(\boldsymbol{T})$ and $(\boldsymbol{K})$ have a similar proportional effect of $(\boldsymbol{B})$. The increase of slip Knudsen number $(\boldsymbol{K} \boldsymbol{n})$ in Figure 4(c) enhances the streamwise velocity near the walls $\left(\boldsymbol{D}_{\text {wall }}\right)$ due to the slipping effect. Whereas, Figure 4(b) discloses that the rise of wall damping coefficient $(\boldsymbol{D})$ resists the motion and causes a reduction in $\left(\boldsymbol{D}_{\text {wall }}\right)$. Figure $4(\mathrm{e})$ is clearly showing the impact of Compressibility number $(\boldsymbol{\chi})$ on $\left(\boldsymbol{D}_{\text {wall }}\right)$ and the results indicate the inversely proportional relation between $(\boldsymbol{\chi})$ and $\left(\boldsymbol{D}_{\text {wall }}\right)$. The rise in the transverse magnetic flux $(\boldsymbol{H a})$ parameter reduces the mean streamwise velocity at walls due to its resistive action; see Figure $4(\mathrm{~g})$. The considered ranges of the governing parameters are taken according to its typical and experimental values in the physical applications related to this case of study. The flow regimes were classified into continuum, slip, transition and free molecular according to Knudsen number ranges. If $\boldsymbol{K n}<\mathbf{0 . 0 0 1}$, the fluid is considered as a continuous medium as a result of neglecting the molecules mean free path compared to the geometrical dimensions. If $\mathbf{0 . 0 0 1}<\boldsymbol{K n}<\mathbf{0 . 1}$, then the fluid tends to slip corresponding to the domain walls. If $\mathbf{0 . 1}<\boldsymbol{K n}<$ 3. 0, so that it is the transition flow regimes. When $\boldsymbol{K n}>$ 3. 0 , the fluid flow exists in the free molecular case. The range of compressibility number is $\mathbf{0} \leq \boldsymbol{\chi} \leq \mathbf{1}$, when $\chi=\mathbf{0}$ the fluid is considered to be incompressible liquid and when $\chi=\mathbf{1}$ case of fully compressible flow exists. The elastic wall parameters ranges are selected according to $[50,51]$.

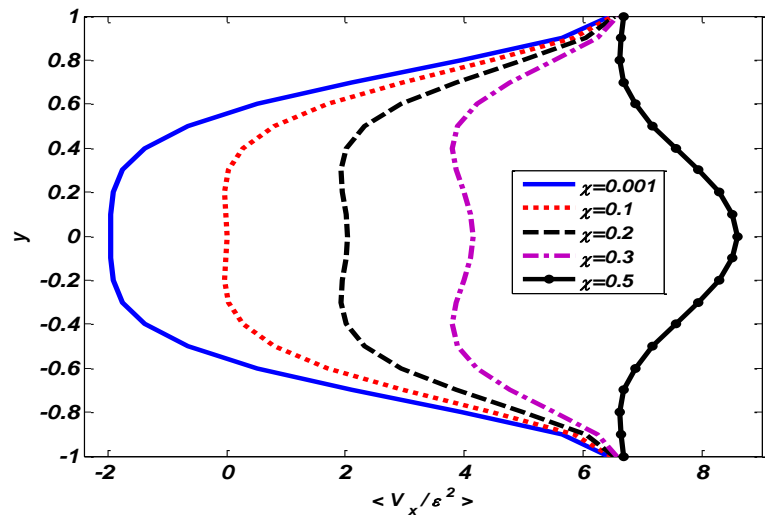

(a) Liquid compressibility effectiveness on the net velocity

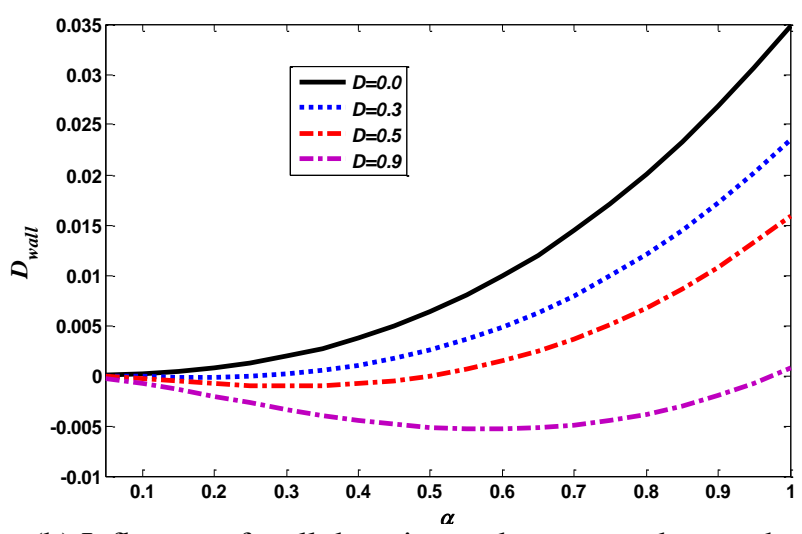

(b) Influence of wall damping and wave number on the velocity at walls 


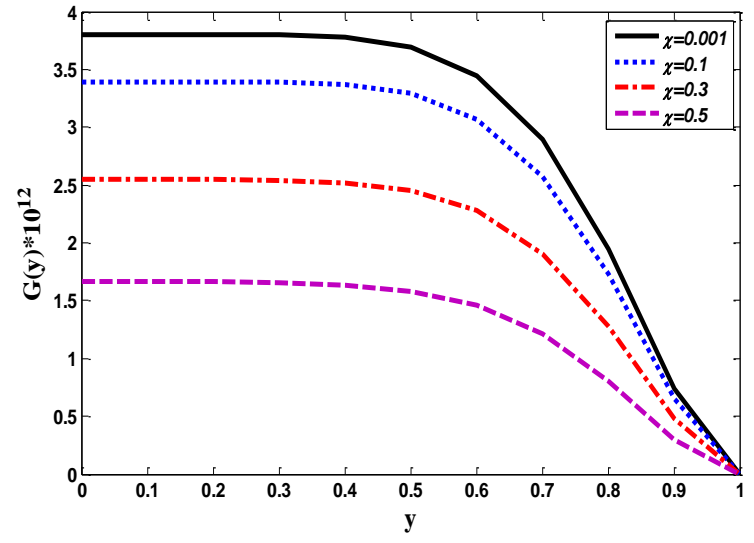

(c) Liquid compressibility effectiveness on the mean velocity perturbation function

Figure 2- Variation of the streamwise velocity at different positions under various values of wall and flow properties

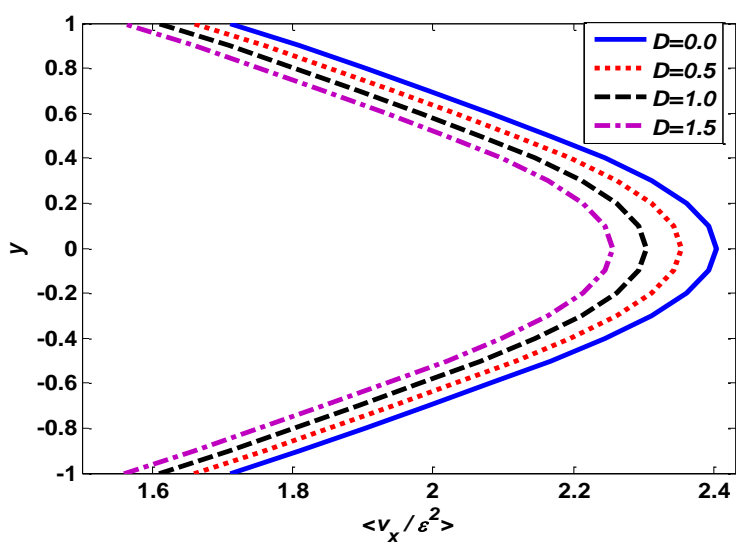

(a ) Influence of wall damping resistance



(b) Influence of the wall spring stiffness

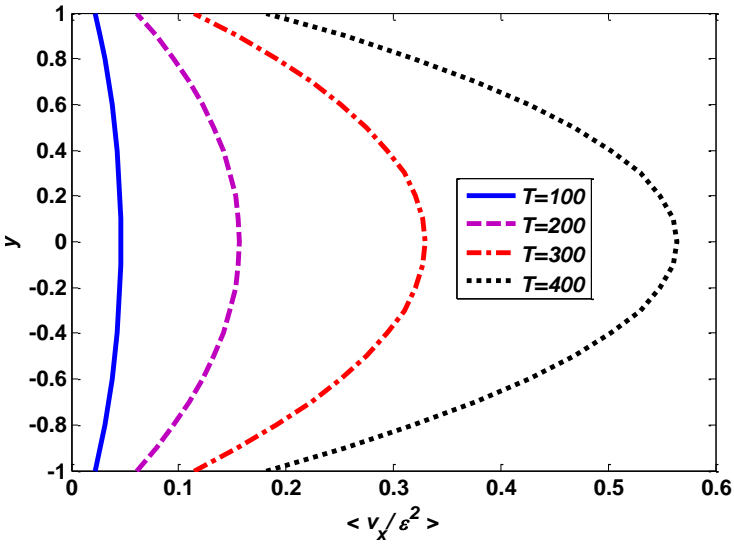

(c) Influence of the longitude wall tension

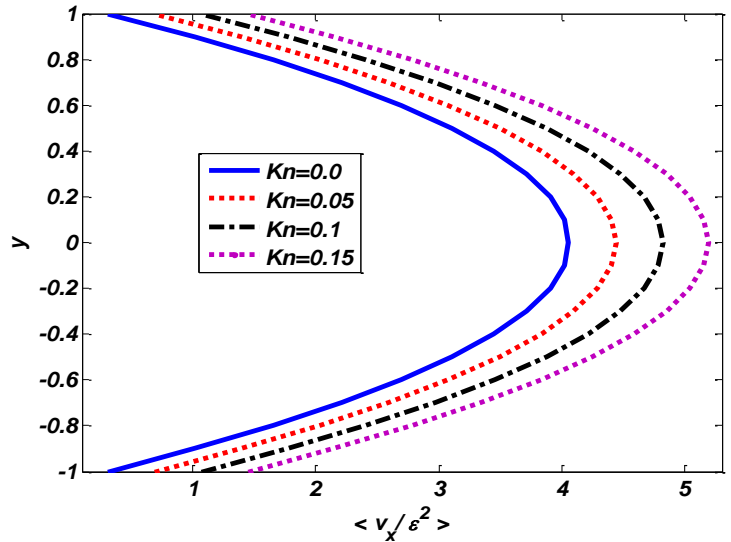

(d) Influence of the slip Knudsen number

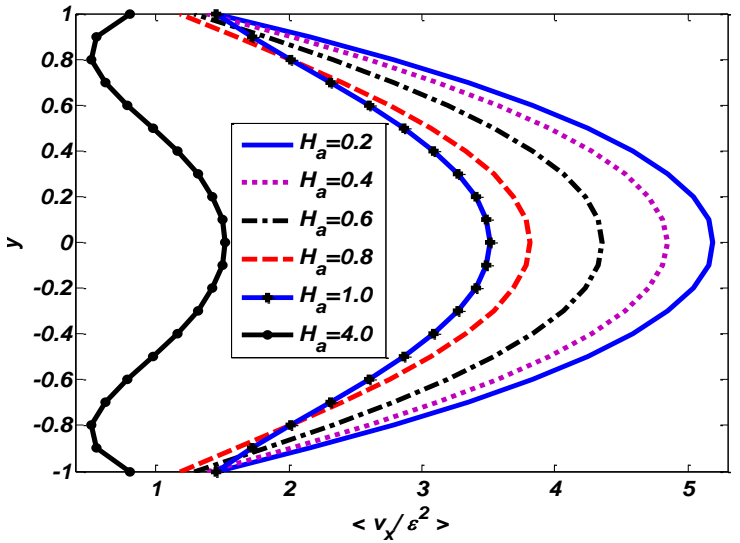

(e) Influence of the magnetic flux Hartmann number

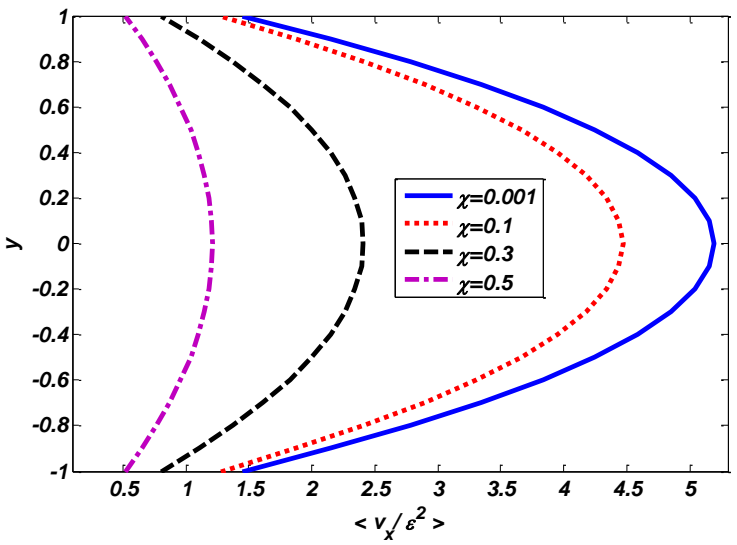

(f) Influence of the liquid compressibility

Figure 3- Variation of the mean streamwise velocity along the $y$ - axis 


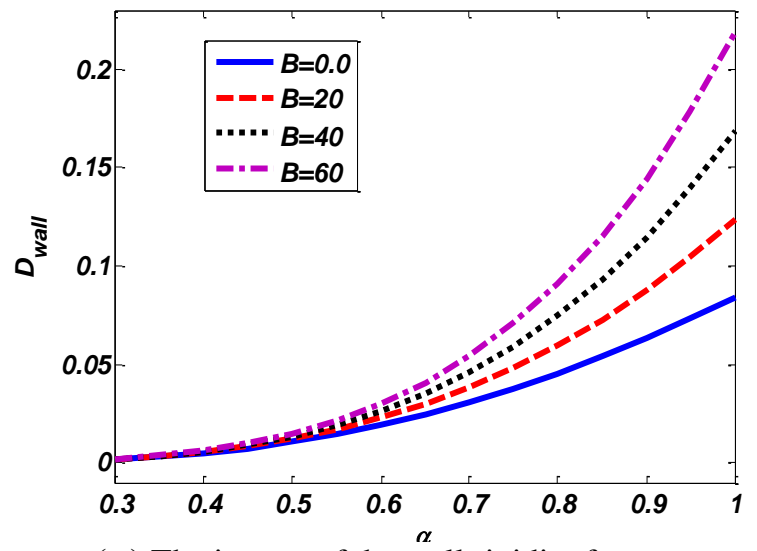

(a ) The impact of the wall rigidity factor

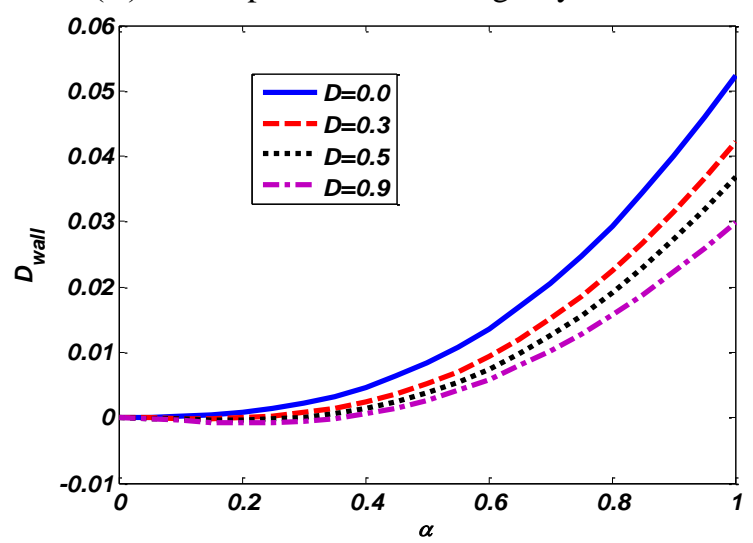

(b) The impact of the wall damping coefficient

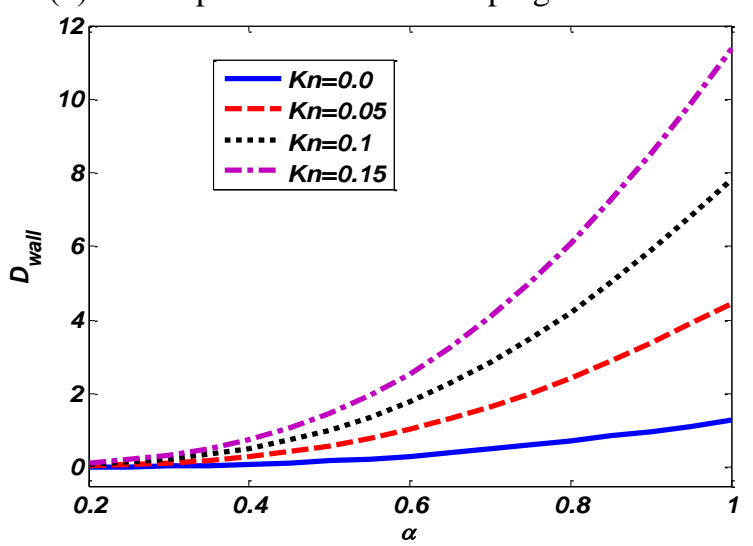

(c) The impact of the wall slip coefficient

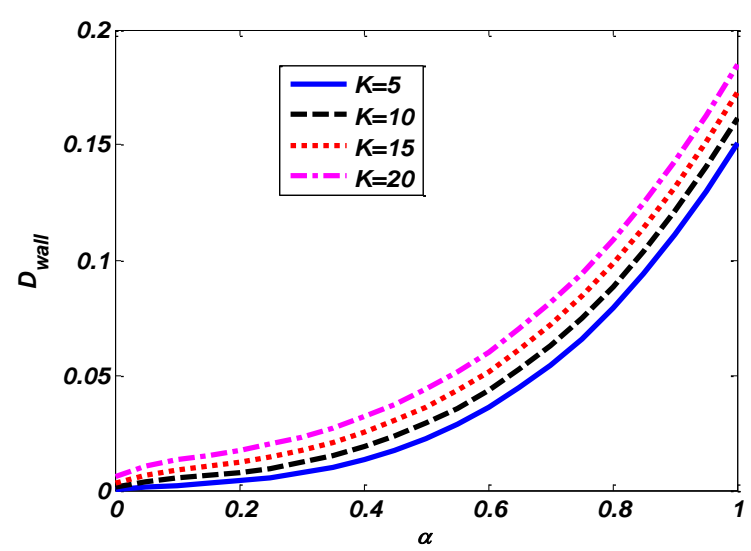

(d) The impact of the wall spring stiffness

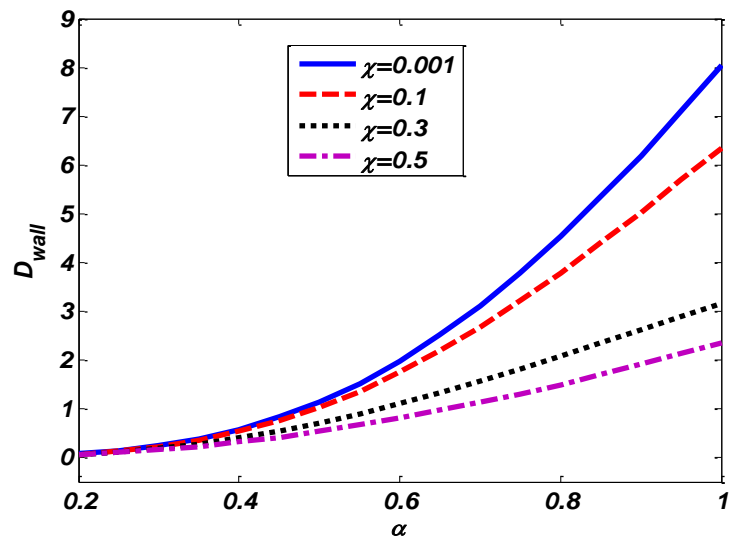

(e) The impact of the compressibility number

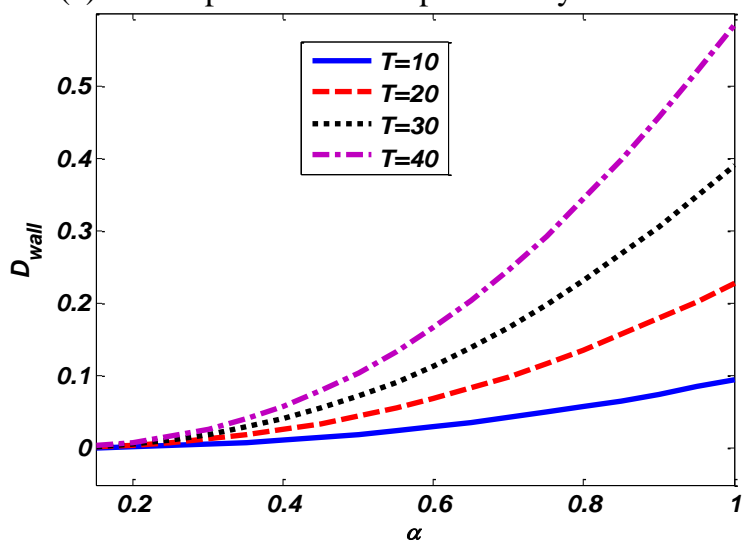

(f) The impact of the wall tension factor

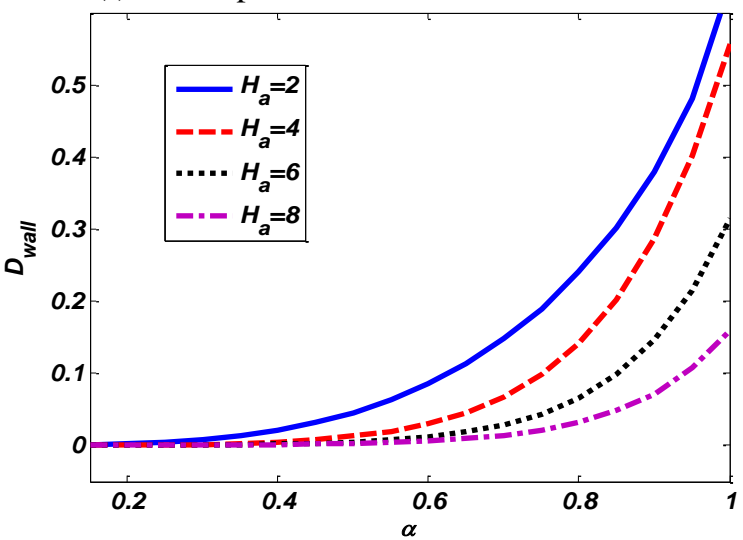

(g) The impact of the magnetic Hartmann number

Figure 4- Variation of the streamwise velocity corresponding to the elastic channel walls versus the wave number 
Table 1- Wall and Flow Parameters in Figure (2)

\begin{tabular}{|c|c|c|c|}
\hline $\begin{array}{c}\text { Wall \& flow } \\
\text { parameters }\end{array}$ & Fig. (a) & Fig. (b) & Fig. (c) \\
\hline $\mathrm{K}$ & 1 & 1 & 0.1 \\
\hline $\mathrm{D}$ & 0.5 & $0-0.3-0.5-0.9$ & 0.01 \\
\hline $\mathrm{T}$ & 9866.7 & 200 & 100 \\
\hline$\chi$ & $0.001-0.1-0.2-0.3-0.5$ & 0.001 & $0.001-0.1-0.3-0.5$ \\
\hline $\mathrm{Kn}$ & 0.0 & 0.1 & 0.0 \\
\hline $\mathrm{B}$ & 2 & 2 & 2 \\
\hline $\mathrm{R}$ & 20 & 100 & 0.5 \\
\hline$\alpha$ & 0.5 & Axis & 0.01 \\
\hline$m$ & 0.01 & 0.01 & 0 \\
\hline $\mathrm{Ha}$ & 0 & 0 & \\
\hline
\end{tabular}

Table 2- Wall and Flow Parameters in Figure (3)

\begin{tabular}{|c|c|c|c|c|c|c|}
\hline $\begin{array}{c}\text { Wall \& } \\
\text { flow } \\
\text { paramet } \\
\text { ers }\end{array}$ & Fig. (a) & Fig. (b) & Fig. (c) & Fig. (d) & Fig. (e) & Fig. (f) \\
\hline D & $\begin{array}{c}0-0.5-1.0- \\
1.5\end{array}$ & 0.1 & 0.1 & 0.5 & 0.5 & 0.5 \\
\hline K & 0.1 & $\begin{array}{c}5-10-15- \\
20\end{array}$ & 5 & 1 & 1 & 1 \\
\hline $\mathrm{T}$ & 200 & 100 & $100-200-300-$ & 500 & 500 & 0.15 \\
\hline $\mathrm{Kn}$ & 0.15 & 0.15 & 0.15 & $0.0-.05-0.1-$ & 0.15 & 0.15 \\
\hline$H a$ & 0.1 & 0.2 & 0.2 & 0.2 & $0.2-0.4-0.6-0.8-$ & $1.0-4.0$ \\
\hline$\chi$ & 0.5 & 0.001 & 0.001 & 0.001 & 0.001 & $0.001-0.1-0.3-$ \\
\hline $\mathrm{R}$ & 10 & 50 & 50 & 20 & 20 & 0.5 \\
\hline$\alpha$ & 0.9 & 0.5 & 0.5 & 0.5 & 0.5 & 20 \\
\hline $\mathrm{B}$ & 20 & 20 & 20 & 2 & 2 & 0.5 \\
\hline$m$ & 0.01 & 0.01 & 0.01 & 0.01 & 0.01 & 0.01 \\
\hline
\end{tabular}

Table 3- Wall and Flow Parameters in Figure (4)

\begin{tabular}{|c|c|c|c|c|c|c|c|}
\hline $\begin{array}{c}\text { Wall \& } \\
\text { flow } \\
\text { paramet } \\
\text { ers }\end{array}$ & Fig. (a) & Fig. (b) & Fig. (c) & Fig. (d) & Fig. (e) & Fig. (f) & Fig. (g) \\
\hline $\mathrm{B}$ & $0.0-20-40-60$ & 2 & 2 & 20 & 2 & 0.2 & 0.2 \\
\hline $\mathrm{D}$ & 0.5 & $\begin{array}{c}0.0-0.3- \\
0.5-0.9\end{array}$ & 0.01 & 0.1 & 0.01 & 0.01 & 0.01 \\
\hline $\mathrm{Kn}$ & 0.15 & 0.15 & $\begin{array}{c}0.0-.05-0.1- \\
0.15\end{array}$ & 0.15 & 0.15 & 0.15 & 0.15 \\
\hline K & 1 & 1 & 0.2 & $\begin{array}{c}\text { 5-10-15- } \\
20\end{array}$ & 1 & 0.2 & 0.2 \\
\hline$\chi$ & 0.001 & 0.001 & 0.001 & 0.001 & $\begin{array}{c}0.001-0.1-0.3- \\
0.5\end{array}$ & 0.001 & 0.001 \\
\hline $\mathrm{T}$ & 100 & 200 & 20 & 100 & 200 & $\begin{array}{c}10-20-30- \\
40\end{array}$ & 40 \\
\hline $\mathrm{Ha}$ & 0.2 & 0.2 & 0.2 & 0.2 & 0.2 & 0.2 & $2-4-6-8$ \\
\hline $\mathrm{R}$ & 50 & 100 & 1 & 50 & 10 & 10 & 10 \\
\hline $\mathrm{m}$ & 0.01 & 0.01 & 0.01 & 0.01 & 0.01 & 0.01 & 0.01 \\
\hline
\end{tabular}




\section{Conclusions}

The principle goal of this study is concerned with the combined interaction of magnetic flux $(\mathrm{Ha})$, the slip conditions $(\mathrm{Kn})$, liquid compressibility $(\chi)$ and compliant elastic wall characteristics $(D, T, K$ and $B)$ on the mean axial streamwise velocity $\left\langle\frac{V_{x}}{\varepsilon^{2}}>\right.$ and the mean streamwise velocity corresponding to elastic channel walls $\left(D_{\text {wall }}\right)$. The relation describing the flow behavior such as pressure gradient, mean axial streamwise velocity and the net flow flux is derived from the governing continuity and momentum equations by using the perturbation methodology with small perturbation amplitude ratio. The results disclose that the magnetic field flux is resisting the flow motion and cause a reduction in the flow flux. The raise in magnetic flux results in a decrease in the mean axial streamwise velocity. For higher values of magnetic flux, the reverse flow is appearing at the core of the channel. And near the boundaries, a similar effect is obtained. The wall damping has also a drag effect on the flow motion and near the walls while the spring stiffness and wall tension are boosting the net flow rate and the mean axial velocity. The slipping at the walls also enhances the net streamwise velocity $\left\langle\frac{V_{x}}{\varepsilon^{2}}>\right.$ and $\left(D_{\text {wall }}\right)$ .on contrast the liquid compressibility $(\chi)$ has a strong action in reducing $<\frac{V_{x}}{\varepsilon^{2}}>$ and $\left(D_{\text {wall }}\right)$.

\section{Nomenclature}

$\begin{array}{clcl}2 d & \text { Channel mean width. } & D & \text { Damping parameter. } \\ \vec{V} & \text { The fluid velocity vector. } & B & \text { Wall bending coefficient. } \\ p & \text { The fluid pressure. } & K & \text { Spring stiffness. } \\ \rho & \text { The fluid density. } & p_{o} & \text { The out-surface pressure and } \\ & & \text { considered to be zero. } \\ x & \text { The streamwise Cartesian direction. } & R & \text { Mean free path of molecules. } \\ y & \text { The normal Cartesian direction. } & \chi & \text { The compressibility number. } \\ t & \text { The time. } & \alpha & \text { The wave number. } \\ u & \text { The fluid velocity in the } x \text {-direction. } & \varepsilon & \text { The amplitude ratio. } \\ v & \text { The fluid velocity in the } y \text {-direction. } & K n & \text { Knudsen number. } \\ \mu & \text { The fluid dynamic viscosity. } & v_{o} & \text { Kinematic viscosity. } \\ K^{*} & \text { The liquid compressibility factor. } & V_{x}(y) & \text { The net axial velocity. } \\ \rho_{o} & \text { The standard density. } & G(y) & \text { The mean velocity perturbation } \\ \eta(x, t) & \text { The vertical wall displacement. } & B_{o} & \text { The magnetic flux at the origin. } \\ a & \text { The wave amplitude. } & \sigma & \text { Electrical conductivity. } \\ \lambda & \text { The wavelength. } & H_{a} & \text { Hartmann number. } \\ c & \text { The wave speed. } & \vec{F} & \text { Electromagnetic force. } \\ L & \text { Compliant wall differential operator. } & <Q> & \text { The net flow rate. } \\ T & \text { Longitudinal tension per unit width } & & \end{array}$




\section{Appendix}

$R=\frac{c d \rho_{0}}{\mu}$

$\chi=k^{*} \rho_{0} c^{2}$

$\alpha=\frac{2 \pi d}{\lambda}$

$\varepsilon=\frac{a}{d}$

$H_{a}=\sqrt{\frac{\sigma}{\mu}} B_{o} d$

$v=\frac{\mu}{\rho_{o}}$

$K n=\frac{A}{d}$

$\delta_{b}=\frac{D \alpha^{2}}{R}$

$\delta_{a}=m \alpha^{3}-\frac{B \alpha^{5}}{R^{2}}-\frac{T \alpha^{3}}{R^{2}}-\frac{K \alpha}{R^{2}}$

$L_{1}^{2}=\frac{Z+\sqrt{Z^{2}-4 \Gamma^{2} v^{2}}}{2}$

$A_{1}=\chi\left(\frac{L_{1}^{2}-\Gamma^{2}}{L_{1} \gamma}\right)+\frac{i L_{1}}{\alpha}$

$A_{2}=\chi\left(\frac{L_{2}^{2}-\Gamma^{2}}{L_{2} \gamma}\right)+\frac{i L_{2}}{\alpha}$

$R_{4}=R_{8}=\left(\frac{L_{1}^{2}-\Gamma^{2}}{L_{1} \gamma}\right)+\frac{R_{1}}{R_{3}}$

$R_{6}=\left[\frac{\chi R_{1}}{R_{3}}+A_{1}\right]$

$R_{10}=\left[\frac{-R_{4} L_{1}}{-i \alpha}\right]$

$w=\left[\frac{4}{3} \alpha^{2}-i \alpha R+H_{a}^{2}\right]$

$g_{2}=\cosh L_{2}+K_{n} L_{2} \sinh L_{2}$

$g_{4}=\left(R_{7} L_{2}^{2}-w R_{7}+i \alpha L_{2}\right) \cosh L_{2}$

$R_{3}=\left[\gamma-\frac{i \beta^{2} \chi}{\alpha}\right]$

$R_{5}=R_{9}=\left(\frac{L_{2}^{2}-\Gamma^{2}}{L_{2} \gamma}\right)+\frac{R_{2}}{R_{3}}$

$R_{7}=\left[\frac{\chi R_{2}}{R_{3}}+A_{2}\right]$

$R_{11}=\left[\frac{-R_{5} L_{2}}{-i \alpha}\right]$

$g_{1}=\cosh L_{1}+K_{n} L_{1} \sinh L_{1}$

$g_{3}=\left(R_{6} L_{1}^{2}-w R_{6}+i \alpha L_{1}\right) \cosh L_{1}$
$G=R_{6} g_{1} g_{4}-R_{7} g_{2} g_{3}$

$C_{2}=R \delta \frac{R_{7} g_{1}}{G}$

$C_{1}=-R \delta \frac{R_{7} g_{2}}{G}$

$\beta_{1}=\frac{1}{2}\left(U_{f 1}^{\prime}(1)+{\overline{U_{f 1}(1)}}^{\prime}\right)$

$\beta_{3}=\frac{1}{2}\left(U_{f 1}^{\prime \prime}(1)+\overline{U_{f 1}(1)}{ }^{\prime \prime}\right)$

$\delta_{1}=\overline{R_{6}} \overline{C_{1}} C_{1} L_{1}+\overline{L_{1}} \overline{C_{1}} C_{1} R_{6}$

$\delta_{3}=\overline{R_{6}} \overline{C_{1}} C_{2} L_{2}+\overline{L_{1}} \overline{C_{1}} C_{2} R_{7}$

$\delta_{5}=\overline{C_{1}} R_{6} C_{1} L_{1}+\overline{L_{1}} \overline{C_{1}} \overline{R_{6}} C_{1}$

$\delta_{7}=\overline{C_{2}} R_{1} C_{1} L_{1}+\overline{L_{1}} \overline{C_{1}} \overline{R_{6}} C_{1}$

$\delta=\frac{-i \alpha}{2 R^{2}}\left[R^{2} m \alpha^{2}-B \alpha^{4}-T \alpha^{2}+i D \alpha R-K\right]$

$\Gamma^{2}=\beta^{2}-H_{a}^{2}$

$\beta^{2}=\left[\alpha^{2}+H_{a}^{2}-i \alpha R\right]$

$S=\delta_{c}=H_{a}^{2}$

$\gamma=R-\frac{i \alpha \chi}{3}$

$B_{1}=1-\frac{i \alpha \chi}{\gamma}$

$Z=v^{2}+\Gamma^{2}+\frac{S}{B_{1}}$

$v^{2}=\beta^{2}-\frac{\beta^{2}-\alpha^{2}}{B_{1}}$

$L_{2}^{2}=\frac{Z-\sqrt{Z^{2}-4 \Gamma^{2} v^{2}}}{2}$

$R_{1}=\left[-\left(\frac{L_{1}^{2}-\Gamma^{2}}{L_{1}}\right)-\frac{i A_{1} L_{1}^{2}}{\alpha}+\frac{i A_{1} \beta^{2}}{\alpha}\right]$

$R_{2}=\left[-\left(\frac{L_{2}^{2}-\Gamma^{2}}{L_{2}}\right)-\frac{i A_{2} L_{2}^{2}}{\alpha}+\frac{i A_{2} \beta^{2}}{\alpha}\right]$

$\beta_{2}=\frac{-1}{2}\left(U_{f 1}^{\prime}(-1)+\overline{U_{f 1}(-1)^{\prime}}\right)$

$\left.\beta_{4}=\frac{-1}{2}\left(U_{f 1}^{\prime \prime}(-1)+\overline{U_{f 1}(-1)}\right)^{\prime \prime}\right)$

$\delta_{2}=\overline{R_{7}} \overline{C_{2}} C_{1} L_{1}+\overline{L_{2}} \overline{C_{2}} C_{1} R_{6}$

$\delta_{4}=\overline{R_{7}} \overline{C_{2}} C_{2} L_{2}+\overline{L_{2}} \overline{C_{2}} C_{2} R_{7}$

$\delta_{6}=\overline{C_{1}} R_{7} C_{2} L_{2}+\overline{L_{1} C_{1}} \overline{R_{6}} C_{2}$

$\delta_{8}=\overline{C_{2}} R_{7} C_{2} L_{2}+\overline{L_{2}} \overline{C_{2}} \overline{R_{7}} C_{2}$

$\beta_{2}=\frac{-1}{2}\left(U_{f 1}^{\prime}(-1)+\overline{U_{f 1}(-1)^{\prime}}\right)$

$D_{3}=-\frac{1}{2\left(\operatorname{Sinh} \sqrt{\delta_{c}}+K n \sqrt{\delta_{c}} \operatorname{Cosh} \sqrt{\delta_{c}}\right)}\left\{R[E(1)-E(-1)]+K n\left[R E^{\prime}(1)+R E^{\prime}(-1)+\beta_{3}+\beta_{4}\right]+\beta_{1}-\beta_{2}\right\}$ 


\section{References}

[1] T. W. Latham, "Fluid motions in a peristaltic pump," Massachusetts Institute of Technology, 1966.

[2] Y. Fung and C. Yih, "Peristaltic transport," Journal of Applied Mechanics, vol. 35, no. 4, pp. 669-675, 1968.

[3] Y.-c. Fung, Biomechanics: mechanical properties of living tissues. Springer Science \& Business Media, 2013.

[4] M. R. Ricard and Y. R. Nuñez, "STABILITY OF LONG WAVE PERISTALTIC TRANSPORT OF COMPRESSIBLE VISCOUS FLUID," in Proc. of the 2006 International Symposium on Mathematical and Computational Biology: BIOMAT 2006, 2006: Editora E-papers.

[5] A. H. Shapiro, M. Y. Jaffrin, and S. L. Weinberg, "Peristaltic pumping with long wavelengths at low Reynolds number," Journal of fluid mechanics, vol. 37, no. 4, pp. 799-825, 1969.

[6] A. Sinha, G. Shit, and N. Ranjit, "Peristaltic transport of MHD flow and heat transfer in an asymmetric channel: Effects of variable viscosity, velocity-slip and temperature jump," Alexandria Engineering Journal, vol. 54, no. 3, pp. 691-704, 2015.

[7] S. Srinivas, R. Gayathri, and M. Kothandapani, "The influence of slip conditions, wall properties and heat transfer on MHD peristaltic transport," Computer Physics Communications, vol. 180, no. 11, pp. 21152122, 2009.

[8] L. Srivastava and V. Srivastava, "On two-phase model of pulsatile blood flow with entrance effects," Biorheology, vol. 20, no. 6, pp. 761-777, 1983.

[9] L. Srivastava and V. Srivastava, "Peristaltic transport of blood: Casson model-II," Journal of Biomechanics, vol. 17, no. 11, pp. 821-829, 1984.

[10] L. Srivastava and V. Srivastava, "Interaction of peristaltic flow with pulsatile flow in a circular cylindrical tube," Journal of biomechanics, vol. 18, no. 4, pp. 247-253, 1985.

[11] L. Srivastava and V. Srivastava, "Peristaltic transport of a particle-fluid suspension," Journal of biomechanical engineering, vol. 111, no. 2, pp. 157$165,1989$.

[12] V. Srivastava and M. Saxena, "A two-fluid model of non-Newtonian blood flow induced by peristaltic waves," Rheologica Acta, vol. 34, no. 4, pp. 406-414, 1995.

[13] V. Srivastava and L. Srivastava, "Effects of Poiseuille flow on peristaltic transport of a particulate suspension," Zeitschrift für angewandte Mathematik und Physik ZAMP, vol. 46, no. 5, pp. 655-679, 1995.

[14] V. Sud, G. Sekhon, and R. Mishra, "Pumping action on blood by a magnetic field," Bulletin of mathematical biology, vol. 39, no. 3, pp. 385-390, 1977.

[15] S. I. Abdelsalam and K. Vafai, "Combined effects of magnetic field and rheological properties on the peristaltic flow of a compressible fluid in a microfluidic channel," European Journal of Mechanics-B/Fluids, vol. 65, pp. 398-411, 2017.

[16] I. Eldesoky, "Influence of slip condition on peristaltic transport of a compressible Maxwell fluid through porous medium in a tube," International Journal of Applied Mathematics and Mechanics, vol. 8, no. 2, pp. 99-117, 2012.

[17] N. S. Akbar, "Application of Eyring-Powell fluid model in peristalsis with nano particles," Journal of Computational and Theoretical Nanoscience, vol. 12, no. 1, pp. 94-100, 2015.

[18] T. Hayat, N. Saleem, Y. Abd Elmaboud, and S. Asghar, "Peristaltic flow of a second-order fluid in the presence of an induced magnetic field," International Journal for Numerical Methods in Fluids, vol. 67, no. 5, pp. 537-558, 2011.

[19] F. Abbasi, T. Hayat, A. Alsaedi, and B. Ahmed, "Soret and Dufour effects on peristaltic transport of MHD fluid with variable viscosity," Applied Mathematics \& Information Sciences, vol. 8, no. 1, p. 211, 2014.

[20] K. S. Mekheimer, S. R. Komy, and S. I. Abdelsalam, "Simultaneous effects of magnetic field and space porosity on compressible Maxwell fluid transport induced by a surface acoustic wave in a microchannel," Chinese Physics B, vol. 22, no. 12, p. 124702, 2013.

[21] Y. A. Elmaboud, S. I. Abdelsalam, K. S. Mekheimer, and K. Vafai, "Electromagnetic flow for two-layer immiscible fluids," Engineering Science and Technology, an International Journal, 2018.

[22] S. R. El Koumy, I. B. El Sayed, and S. I. Abdelsalam, "Hall and porous boundaries effects on peristaltic transport through porous medium of a Maxwell model," Transport in porous media, vol. 94, no. 3, pp. 643-658, 2012.

[23] S. I. Abdelsalam and K. Vafai, "Particulate suspension effect on peristaltically induced unsteady pulsatile flow in a narrow artery: blood flow model," Mathematical biosciences, vol. 283, pp. 91-105, 2017.

[24] S. I. Abdelsalam and M. M. Bhatti, "The impact of impinging $\mathrm{TiO} 2$ nanoparticles in Prandtl nanofluid along with endoscopic and variable magnetic field effects on peristaltic blood flow," Multidiscipline Modeling in Materials and Structures, 2018.

[25] S. I. Abdelsalam and M. Bhatti, "The study of nonNewtonian nanofluid with hall and ion slip effects on peristaltically induced motion in a non-uniform channel," RSC advances, vol. 8, no. 15, pp. 79047915, 2018.

[26] Y. Abd Elmaboud, K. S. Mekheimer, and S. I. Abdelsalam, "A study of nonlinear variable viscosity in finite-length tube with peristalsis," Applied Bionics and Biomechanics, vol. 11, no. 4, pp. 197-206, 2014.

[27] A. Aarts and G. Ooms, "Net flow of compressible viscous liquids induced by travelling waves in porous media," Journal of engineering mathematics, vol. 34, no. 4, pp. 435-450, 1998. 
[28] I. M. Eldesoky and A. A. Mousa, "Peristaltic pumping of fluid in cylindrical tube and its applications in the field of aerospace," in 13th International Conference on Aerospace Sciences and Aviation Technology, Military Technical College, Cairo, 2009, pp. 1-14.

[29] I. M. Eldesoky and A. Mousa, "Peristaltic flow of a compressible non-Newtonian Maxwellian fluid through porous medium in a tube," International Journal of Biomathematics, vol. 3, no. 02, pp. 255275, 2010.

[30] I. Eldesoky, S. Abdelsalam, R. Abumandour, M. Kamel, and K. Vafai, "Interaction between compressibility and particulate suspension on peristaltically driven flow in planar channel," Applied Mathematics and Mechanics, vol. 38, no. 1, pp. 137154, 2017.

[31] R. M. Abumandour, I. Eldesoky, and F. A. Kroush, "Effects of slip conditions and compressibility on the peristaltic flow of particulate suspension in a planar channel," SN Applied Sciences, vol. 1, no. 10, p. 1305, 2019.

[32] S. Pandey and M. Chaube, "Study of wall properties on peristaltic transport of a couple stress fluid," Meccanica, vol. 46, no. 6, pp. 1319-1330, 2011.

[33] M. Javed, T. Hayat, and A. Alsaedi, "Effect of wall properties on the peristaltic flow of a non-Newtonian fluid," Applied Bionics and Biomechanics, vol. 11, no. 4, pp. 207-219, 2014.

[34] M. A. A. Elnaby and M. H. Haroun, "A new model for study the effect of wall properties on peristaltic transport of a viscous fluid," Communications in Nonlinear Science and Numerical Simulation, vol. 13, no. 4, pp. 752-762, 2008.

[35] I. M. Eldesoky, R. M. Abumandour, and E. T. Abdelwahab, "Analysis for various effects of relaxation time and wall properties on compressible Maxwellian peristaltic slip flow," Zeitschrift für Naturforschung A, vol. 74, no. 4, pp. 317-331, 2019.

[36] I. Eldesoky, R. Abumandour, M. Kamel, and E. Abdelwahab, "The combined influences of heat transfer, compliant wall properties and slip conditions on the peristaltic flow through tube," $S N$ Applied Sciences, vol. 1, no. 8, p. 897, 2019.

[37] K. S. Mekheimer and A. J. N. M. f. P. D. E. AbdelWahab, "Effect of wall compliance on compressible fluid transport induced by a surface acoustic wave in a microchannel," vol. 27, no. 3, pp. 621-636, 2011.

[38] H. Sadaf and S. I. Abdelsalam, "Adverse effects of a hybrid nanofluid in a wavy non-uniform annulus with convective boundary conditions," RSC Advances, vol. 10, no. 26, pp. 15035-15043, 2020.

[39] M. M. Bhatti, M. Marin, A. Zeeshan, R. Ellahi, and S. I. Abdelsalam, "Swimming of Motile Gyrotactic Microorganisms and Nanoparticles in Blood Flow
Through Anisotropically Tapered Arteries," Frontiers in Physics, vol. 8, p. 95, 2020.

[40] S. Abdelsalam and M. Bhatti, "Anomalous reactivity of thermo-bioconvective nanofluid towards oxytactic microorganisms," Appl. Math. Mech, 2020.

[41] Y. A. Elmaboud, S. I. Abdelsalam, K. S. Mekheimer, and K. Vafai, "Electromagnetic flow for two-layer immiscible fluids," Engineering Science and Technology, an International Journal, vol. 22, no. 1, pp. 237-248, 2019.

[42] S. I. Abdelsalam and K. S. Mekheimer, "Couple stress fluid flow in a rotating channel with peristalsis," Journal of Hydrodynamics, vol. 30, no. 2, pp. 307-316, 2018.

[43] I. Eldesoky, S. Abdelsalam, W. El-Askary, A. ElRefaey, and M. Ahmed, "Joint effect of thermal energy and magnetic field on particulate fluid suspension in a catheterized tube," Bionanoscience, vol. 9, no. 3, pp. 723-739, 2019.

[44] I. Eldesoky, S. I. Abdelsalam, W. El-Askary, and M. Ahmed, "Concurrent Development of Thermal Energy with Magnetic Field on a Particle-Fluid Suspension Through a Porous Conduit," BioNanoScience, vol. 9, no. 1, pp. 186-202, 2019.

[45] S. I. Abdelsalam and M. Bhatti, "New insight into AuNP applications in tumour treatment and cosmetics through wavy annuli at the nanoscale," Scientific reports, vol. 9, no. 1, pp. 1-14, 2019.

[46] M. Sohail, R. Naz, and S. I. Abdelsalam, "Application of non-Fourier double diffusions theories to the boundary-layer flow of a yield stress exhibiting fluid model," Physica A: Statistical Mechanics and its Applications, vol. 537, p. 122753, 2020.

[47] M. Sohail, R. Naz, and S. I. Abdelsalam, "On the onset of entropy generation for a nanofluid with thermal radiation and gyrotactic microorganisms through 3D flows," Physica Scripta, vol. 95, no. 4, p. 045206, 2020.

[48] S. I. Abdelsalam, M. Bhatti, A. Zeeshan, A. Riaz, and O. A. Bég, "Metachronal propulsion of a magnetised particle-fluid suspension in a ciliated channel with heat and mass transfer," Physica Scripta, vol. 94, no. 11, p. 115301, 2019.

[49] S. Elkoumy, E. Barakat, and S. Abdelsalam, "Hall and transverse magnetic field effects on peristaltic flow of a Maxwell fluid through a porous medium," Global J. Pure Appl. Math, vol. 9, no. 2, pp. 187-203, 2013.

[50] V. Shankar and V. Kumaran, "Stability of nonparabolic flow in a flexible tube," Journal of Fluid Mechanics, vol. 395, pp. 211-236, 1999.

[51] V. Shankar and V. Kumaran, "Stability of wall modes in fluid flow past a flexible surface," Physics of fluids, vol. 14, no. 7, pp. 2324-2338, 2002. 